\title{
Chronic hypoventilation and its management
}

\author{
Anita K. Simonds
}

Affiliations: NIHR Respiratory Biomedical Research Unit, Royal Brompton and Harefield NHS Foundation Trust, London, UK.

Correspondence: A.K. Simonds, NIHR Respiratory Biomedical Research Unit, Royal Brompton and Harefield NHS Foundation Trust, London, SW3 6NP, UK. E-mail: A.Simondsarbht.nhs.uk

ABSTRACT While obstructive sleep apnoea syndrome dominates discussion of the prevalence of sleep disordered breathing, nocturnal hypoventilation remains extremely prevalent in those with chronic ventilatory disorders and in the natural history of these conditions pre-dates the development of daytime ventilatory failure. In this review the clinical management of chronic hypoventilation in neuromuscular disease will be considered and then compared with that in obesity hypoventilation syndrome. In simple terms these conditions illustrate the polar opposite ends of the spectrum, as in neuromuscular disease the reduced capacity of the respiratory system is unable to withstand a normal respiratory load, and in obesity hypoventilation syndrome the normal capacity of the respiratory system is unable to tolerate a substantially increased ventilatory load.

O

@ERSpublications

Clinical management of chronic hypoventilation in neuromuscular disease and obesity hypoventilation syndrome http://ow.ly/n2yy9

\section{Neuromuscular disease}

Appropriate clinical management is shown in table 1.

\section{Anticipate high risk conditions}

Experience over the past 10-20 years and collaboration between neurology and respiratory teams has now enabled the identification of many inherited neuromuscular conditions associated with a high, medium and low risk of respiratory decompensation see table 2 [1]. For example, in spinal muscular atrophy (SMA) type 1 all children will develop respiratory failure by the age of 2 years unless treated and around $40 \%$ of those with SMA type 2 develop respiratory failure in childhood. In Duchenne muscular dystrophy respiratory decompensation tends to follow loss of ambulation and $\sim 70 \%$ of patients with Ullrich congenital muscular dystrophy will experience respiratory failure in adolescence. Other conditions with very early onset respiratory failure include those with X-linked myotubular myopathy and those with SMARD (SMA with respiratory distress) [2]. There are important caveats: patients with rigid spine syndrome may develop respiratory failure while still ambulant with relatively preserved vital capacity; and children with congenital myasthenia may experience severe ventilatory failure during acute apnoeic episodes, despite previous normal diurnal arterial blood gas tensions and no evidence of sleep disordered breathing.

\section{Assessment}

Clinical review of respiratory health status is an important part of every consultation for patients with neuromuscular disease, particularly those at high and medium risk of deterioration. Vital capacity should be measured in all those capable of spirometry. Arm span or ulnar length can be used to predict normal values

Received: May 152013 | Accepted after revision: July 112013

Conflict of interest: Disclosures can be found alongside the online version of this article at err.ersjournals.com

Provenance: Submitted article, peer reviewed.

Copyright @ERS 2013 


\section{TABLE 1 Appropriate clinical management for neuromuscular disease}

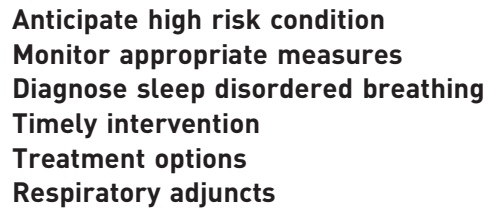

in those with scoliosis, where true height it is difficult to establish. Secretion clearance can be a notable problem before the development of respiratory failure in those in whom expiratory muscle weakness outstrips inspiratory muscle weakness, e.g. SMA type 2, but is also a problem in those with both inspiratory and expiratory muscle weakness, e.g. Duchenne muscular dystrophy. Cough peak flow can be used to establish cough efficiency. Values of $<270 \mathrm{~L} \cdot \mathrm{min}^{-1}$ when well in an adult or adolescent suggest problems with secretion clearance will occur during a chest infection (in which muscle strength will fall further) and values below $160 \mathrm{~L} \cdot \mathrm{min}^{-1}$ suggest cough is profoundly weak. Cough peak flow can be measured in the clinic using a standard peak flow meter. Spot arterial oxygen saturation measured by pulse oximetry $\left(S_{\mathrm{pO}_{2}}\right)$ is helpful in that values $\leqslant 93 \%$ suggest swift further investigation with chest radiography and measurement of arterial carbon dioxide tension and arterial oxygen tension, but a low $\mathrm{SpO}_{2}$ is of course a late feature of incipient respiratory failure.

\section{Mechanisms of hypoventilation in neuromuscular disease}

Both inspiratory muscle weakness and scoliosis contribute to the restrictive ventilatory defect found in patients with neuromuscular disease. Differences in the pattern of sleep disordered breathing tend to follow from the distribution of respiratory and bulbar muscle involvement. Obstructive apnoea is frequently seen in younger Duchenne muscular dystrophy patients and it is possible that steroid therapy predisposes to this. As diaphragm involvement progresses rapid eye movement (REM)-related hypoventilation is seen, which progresses to persistent hypoventilation during non-REM and REM sleep once vital capacity (VC) falls below $40 \%$ [3]. In those with preserved diaphragm function, e.g. individuals with type 2 SMA, mild hypoxaemia with minimal hypercapnia or normocapnia may be seen overnight. Blunting of hypercapnic ventilatory drive by chronic hypercapnia is part of the vicious cycle, although in some patients even a normal level of ventilatory drive is simply insufficient to maintain normocapnia in the face of chronic inspiratory muscle weakness. NicKOL et al. [4] have shown that noninvasive ventilation (NIV) in patients

\section{TABLE 2 Probability of respiratory decompensation in inherited neuromuscular disorders}

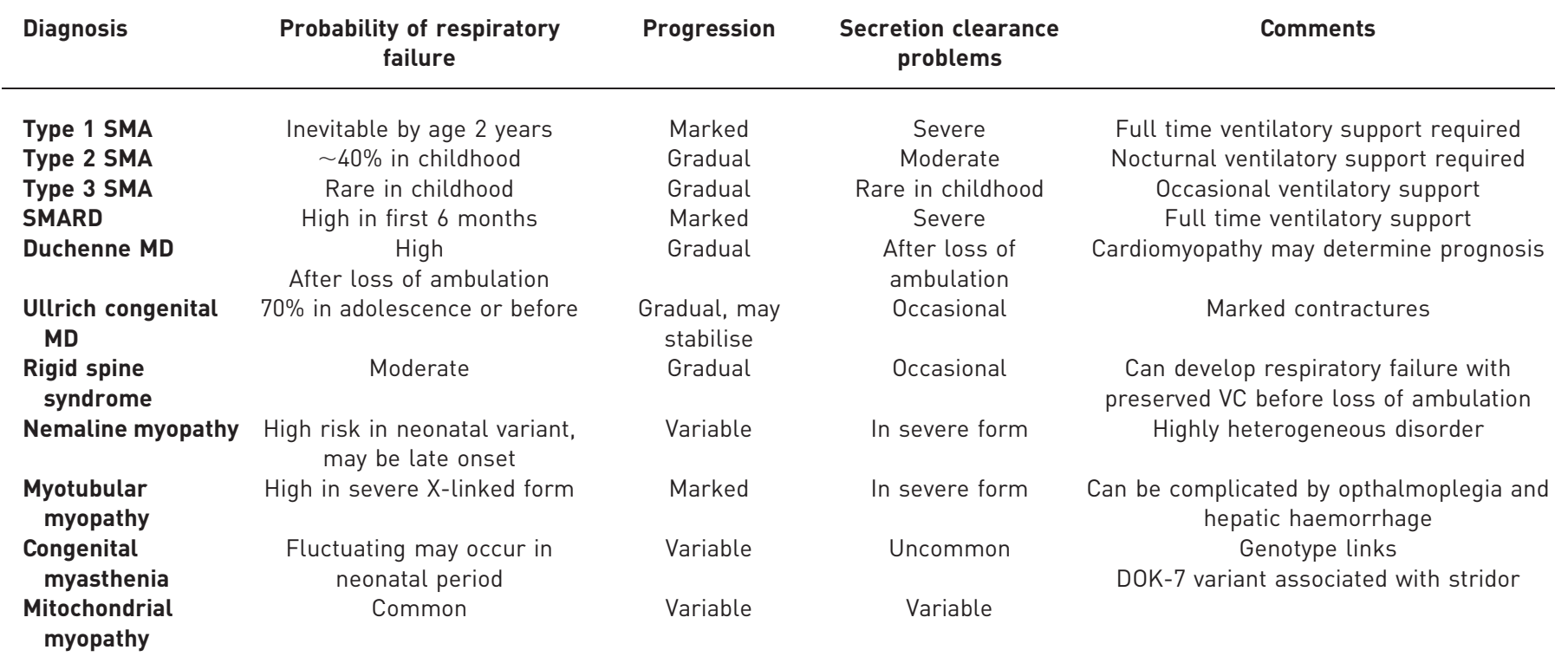

SMA: spinal muscular atrophy; SMARD: SMA with respiratory distress; MD: muscular dystrophy; VC: vital capacity. Reproduced from [1] with permission from the publisher. 
with restrictive disorders due to neuromuscular disease or chest wall disorders is effective at controlling daytime arterial blood gas tensions, as it improves chemosensitivity and has a minimal effect on respiratory muscle strength.

\section{Diagnosis of nocturnal hypoventilation}

There are a number of definitions of nocturnal hypoventilation, for example carbon dioxide tension $\left(\mathrm{PCO}_{2}\right)$ $>50 \mathrm{mmHg}$ for more than $5 \%$ study time, a $10 \mathrm{mmHg}$ rise in carbon dioxide overnight or peak transcutaneous $\mathrm{PCO}_{2}>6.5 \mathrm{kPa}$. Nocturnal hypercapnia has been defined as a mean overnight $P_{\mathrm{CO}_{2}}$ $>50 \mathrm{mmHg}$. The most recent American Academy of Sleep Medicine scoring rules for respiratory events define hypoventilation during sleep as: 1) arterial carbon dioxide (or transcutaneous or end-tidal carbon dioxide as surrogates) $>55 \mathrm{mmHg}$ for $\geqslant 10 \mathrm{~min}$; or 2) there is an increase in $\mathrm{PCO}_{2}$ of $>10 \mathrm{mmHg}$ from the awake supine value to a value exceeding $50 \mathrm{mmHg}$ for $\geqslant 10 \mathrm{~min}$. For those in the paediatric age range hypoventilation is scored when $\mathrm{PCO}_{2}$ is $>50 \mathrm{mmHg}$ for more than $25 \%$ of the total sleep time. Of course it can easily be seen that these values are fairly arbitrary and, for example, the rise in $\mathrm{PCO}_{2}$ by $10 \mathrm{mmHg}$ will be more easily identified in patients with REM-related hypoventilation. Additionally, in the absence of daytime hypercapnia guidelines suggest the introduction of ventilatory support at the onset of symptomatic nocturnal hypoventilation. However, symptoms can be notoriously variable and non-specific (e.g. poor sleep quality, daytime lassitude, morning headaches, anorexia for breakfast, nocturnal sweating or waking with panic sensation) so should be actively sought at each consultation.

The British Thoracic Society guidelines for respiratory management of children with neuromuscular weakness [1] suggest sleep studies should be carried out in patients with symptoms of nocturnal hypoventilation or obstructive sleep apnoea (OSA), and should be undertaken at least once a year in patients with a $\mathrm{VC}<60 \%$ predicted or if patients have lost ambulation in Duchenne muscular dystrophy (table 3). This fits with the findings of RAGETTE et al. [3] who showed a VC of $<60 \%$ predicted was consistent with onset of sleep disordered breathing, at a $\mathrm{VC}<40 \%$ predicted continuous hypoventilation was found and with a VC $<25 \%$ predicted daytime ventilator failure was likely to be present in a mixed group with muscular dystrophies and myopathies. A low threshold for sleep study should also be observed in patients with rigid spine syndrome and diaphragmatic weakness. While some guidelines indicate full polysomnography should be performed it is acknowledged that this is not always possible, so multi-channel respiratory studies can suffice. Actigraphy may be a useful simple guide to sleep-wake cycles and sleep quality in some patients [5].

\section{Indications for noninvasive ventilation}

As discussed above, NIV is indicated in patients with daytime hypercapnia and symptomatic nocturnal hypoventilation in the absence of raised daytime $\mathrm{PCO}_{2}$. This is based on findings that symptoms can be successfully reversed using NIV [6] and on a randomised controlled trial in which patients with nocturnal hypoventilation, but normal daytime arterial blood gas tensions were randomised to NIV or control group [7]. The follow-up involved each group receiving 6-monthly sleep studies for 2 years. NIV was started in the control group if preset criteria were met, e.g. daytime hypercapnia or recurrent chest infections. The trial was limited by the fact that the patient group was heterogeneous, but the outcome was clear cut in that $70 \%$

TABLE 3 Indications for sleep studies in inherited neuromuscular disorders

Indication for sleep study

Comment

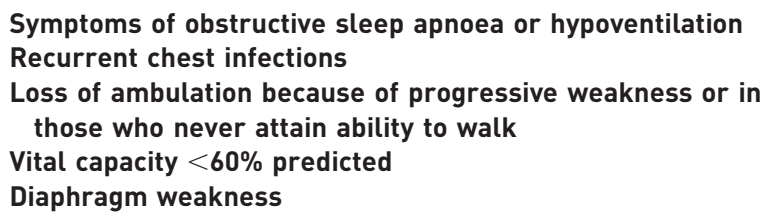

Infantile onset muscle weakness

Rigid spine syndrome

Assessment for surgery or pregnancy feasibility
May indicate nocturnal hypoventilation, consider secretion clearance adjuncts Often a feature in Duchenne muscular dystrophy

Usually bilateral diaphragm weakness required for nocturnal hypoventilation to develop, but unilateral weakness combined with other lung disease may precipitate sleep disordered breathing

Often associated with severe muscle weakness

Nocturnal hypoventilation can occur despite near normal vital capacity

Consider as part of evaluation of patients with neuromuscular disease in addition to pulmonary function tests, measurement of respiratory muscle strength and echocardiography

Reproduced from [1] with permission from the publisher. 
of patients in control group required NIV within 12 months and 90\% within 24 months. Health-related quality of life was improved in those randomised to NIV and several patients in the control group had unplanned admissions for acute ventilatory decompensation. As many inherited neuromuscular conditions are rare and the mechanisms of ventilatory decline are similar it is unlikely that controlled trials will be possible in all diagnostic groups, so most centres now initiate NIV when nocturnal hypoventilation is detected. The advantage is that there is time to prepare both patient and their family for the intervention rather than clandestine initiation at the time of acute ventilatory failure, which is clearly to be avoided.

\section{Motor neurone disease/amyotrophic lateral sclerosis}

As in congenital and inherited neuromuscular disease, described above, monitoring of symptoms and respiratory function and breathing during sleep are key to management of chronic hypoventilation in motor neurone disease/amyotrophic lateral sclerosis (MND/ALS). While VC has the greatest sensitivity and specificity in predicting sleep disordered breathing and ventilatory failure in congenital muscular dystrophies and myopathies, LyaLL et al. [8] have shown that sniff inspiratory pressure more linearly follows the decline in respiratory muscle function in MND/ALS, and once even mild daytime hypercapnia occurs acute ventilator decompensation may be imminent. BOURKe et al. [9] showed that NIV introduced at the time of daytime hypercapnia or orthopnoea resulted in a significant survival advantage of $\sim 7$ months in MND/ALS patients with mild and moderate bulbar involvement, but did not improve prognosis in those with severe bulbar disease at onset. However, even in this severe bulbar group sleep-related symptoms were improved by NIV. Currently, trials investigating the role of diaphragm stimulation in MND/ALS are in progress.

\section{Other interventions}

Low flow long-term oxygen therapy (LTOT) is not recommended in patients with nocturnal hypoventilation as it may worsen hypercapnia but abolish hypoxic ventilatory drive. There are reports of the use of high flow oxygen therapy in children with OSA [10], and in those who fail continuous positive airway pressure (CPAP); however, this is unlikely to be first-line therapy and should be used with caution, and careful monitoring of $\mathrm{PCO}_{2}$. CPAP is used in patients with predominant OSA, but if marked expiratory muscle weakness is present NIV is preferable. CPAP has also been used in patients with mild sleep disordered breathing and type II SMA. Here the main problem initially may be ventilation-perfusion mismatch due to a combination of sputum retention and pulmonary hypoplasia. While CPAP may stabilise respiratory status for a while, progression to nocturnal hypoventilation is likely if inspiratory and expiratory muscle strength decline, hence progression to NIV will be required.

\section{Cough assist devices}

An effective cough requires the presence of a deep inspiration, competent laryngeal function to allow intrathoracic pressure rise and preserved expiratory muscle action, such that an effective force is developed to sheer mucus from the airways and for it to be expectorated. Standard physiotherapy is an important part of the management of adults and children with respiratory muscle weakness and can be performed during NIV which will augment inspiration and reduce tiring. Maximum insufflation capacity is the maximum volume of air stacked in the lungs beyond spontaneous VC. This augmented volume can be achieved by glossopharyngeal breathing in which the patient gulps down air in a rapid sequence or externally using an Ambu bag, which is expelled into the patient's lungs in synchrony with the patient's inspiration. The cough in-exsufflator device provides a large insufflation and then cycles to negative pressure to mimic expiration. A variety of these devices are available which can be used manually or automatically. A randomised comparison with manual physiotherapy and NIV showed that the in-exsufflator device was most effective in increasing cough peak flow in adults and children [11]. The combination of inspiratory muscle assistance with NIV and expiratory muscle assistance with cough insufflation-exsufflation was shown to be more effective than tracheostomy ventilation in reducing pulmonary morbidity in Duchenne muscular dystrophy patients [12] and this noninvasive approach is usually preferred by patients [13].

\section{NIV outcomes}

NIV in Duchenne muscular dystrophy has markedly changed the natural history of the disorder. From a median age at death of 18-20 years, patients using NIV now live to their late twenties $[14,15]$ and around a third may live into their thirties and forties [16]. There are variations in practice. Japanese studies have shown a survival advantage to NIV, but in Scandinavia and some European countries a sequential approach of NIV and then tracheostomy ventilation is used. Comparisons in outcome are difficult as tracheostomy ventilation is applied later in the natural history of Duchenne muscular dystrophy [17] and there have been no randomised trials of invasive versus noninvasive ventilation in Duchenne patients with ventilatory failure.

NIV has also changed the outlook in infants with other conditions, such as type I SMA [18]. While in some cases survival may be significantly increased, in others NIV may be used to palliate symptoms related to 
chronic hypoventilation and facilitate discharge home [19]. Randomised trials are likely to be unethical in type I SMA (except in the very early stages of hypoventilation), so it is difficult to examine outcomes in rare conditions. However, it is clear that the natural history of many conditions has been changed by addressing the vicious cycle which flows from nocturnal hypoventilation. This has major economic implications for healthcare, as increasing numbers of children with neuromuscular disorders are surviving to adulthood and will require long-term follow-up by adult services.

\section{Monitoring the efficacy of NIV}

To ensure optimal outcomes the adherence to NIV and its efficacy overnight should be monitored. Polygraphy or polysomnography can be helpfully combined with downloaded data on adherence, leaks and residual respiratory events. There are recent useful schemas for evaluating the efficacy of NIV [20, 21].

\section{Obesity hypoventilation syndrome}

The definition of obesity hypoventilation syndrome (OHS) is daytime $\mathrm{PCO}_{2}>45 \mathrm{mmHg}$ and oxygen tension $<70 \mathrm{mmHg}$, in the presence of body mass index $>30 \mathrm{~kg} \cdot \mathrm{m}^{-2}$ and sleep disordered breathing, and in the absence of another explanation for hypercapnia. The majority of OHS patients, approximately $80-90 \%$, have OSA but a minority have pure nocturnal hypoventilation with rise in $\mathrm{PCO}_{2}$ by $>10 \mathrm{mmHg}$ at night. Unsurprisingly the prevalence of OHS is increasing as global levels of obesity rise with an estimated prevalence of $0.3-0.4 \%$ in the general population and $10-20 \%$ in those with sleep disordered breathing. However, as noted by PÉPIN et al. [22] OHS is frequently unrecognised until the individual presents with acute on chronic ventilatory decompensation.

\section{Mechanisms of nocturnal hypoventilation in OHS}

The mass load of obesity on respiratory mechanics reduces chest wall compliance and lowers lung volumes, including functional residual capacity. This low lung volume pattern of breathing reduces pulmonary compliance and also leads to expiratory flow limitation in the lower airways generating intrinsic positive end-expiratory pressure and air-trapping. These changes, together with upper airway resistance, further load the inspiratory muscles. As a result higher levels of ventilatory drive are required to maintain normocapnia and chemosensitivity is progressively impaired by rising carbon dioxide level. As pointed out by BERGER et al. [23], this conventional explanation of nocturnal hypoventilation does not fully explain why individuals remain persistently hypercapnic during the day. These authors hypothesise that chronic hypercapnia results from the inability to offload carbon dioxide during the day in response to carbon dioxide loading at night from sleep disordered breathing. Offloading is more likely to occur in those with short episodes of hypercapnia, due to apnoeas and hyponoeas, compared with patients with long periods of hypoventilation (fig. 1). In eucapnic individuals and those with OSA the acute hypercapnia which ensues from a brief apnoea or hyponoea is rapidly reversed by augmented tidal volume in the following few breaths. Modelling work suggests that when apnoea events become more than three-times longer than the breathing interval between them, carbon dioxide will accumulate despite maximum augmentation of tidal volume as there is insufficient time for this hyperventilation to return carbon dioxide to normal before the next episode of carbon dioxide loading [23]. A secondary mechanism which then impairs chemosensitivity is the increase in serum and cerebrospinal fluid bicarbonate, which is exacerbated by reduced renal bicarbonate excretion. It is this combination of mechanical and sleep-related effects that causes chronic alveolar hypoventilation. It is of note that a raised serum bicarbonate level of $>27 \mathrm{mmol} \cdot \mathrm{L}^{-1}$ is a useful screening test for OHS.

Leptin resistance and neurohumeral modulation may play additional roles, which are difficult to quantitate from human studies [23]. However, raised leptin levels are found in OHS patients, probably as a compensatory mechanism to leptin resistance, and these raised levels in turn reduce chemosensitivity to carbon dioxide.

\section{CPAP or NIV in OHS?}

PIPER et al. [24] compared CPAP versus bilevel NIV in OHS patients but excluded those with severe nocturnal desaturation or carbon dioxide rise $>10 \mathrm{mmHg}$. Daytime $\mathrm{PCO}_{2}$ levels decreased similarly in both groups and there was no difference in equipment compliance (mean \pm SD $5.8 \pm 2.4 \mathrm{~h}$ for CPAP; $6.1 \pm 2.1 \mathrm{~h}$ for bilevel NIV), and both groups experienced improvements in sleepiness and psychomotor vigilance. However, for safety reasons it is noteworthy that those patients with more severe hypoventilation who might be less likely to respond to CPAP were excluded.

A further significant problem is management of acute hypercapnic respiratory failure in OHS. CARRILLO et al. [25] carried out an interesting study comparing the use of NIV in acute exacerbations of COPD and OHS. Consecutive patients were treated with a similar NIV protocol based on the finding of respiratory acidosis, dyspnoea and respiratory rate of $\geqslant 25 \cdot \mathrm{min}^{-1}$. Initial settings were an inspiratory positive pressure 


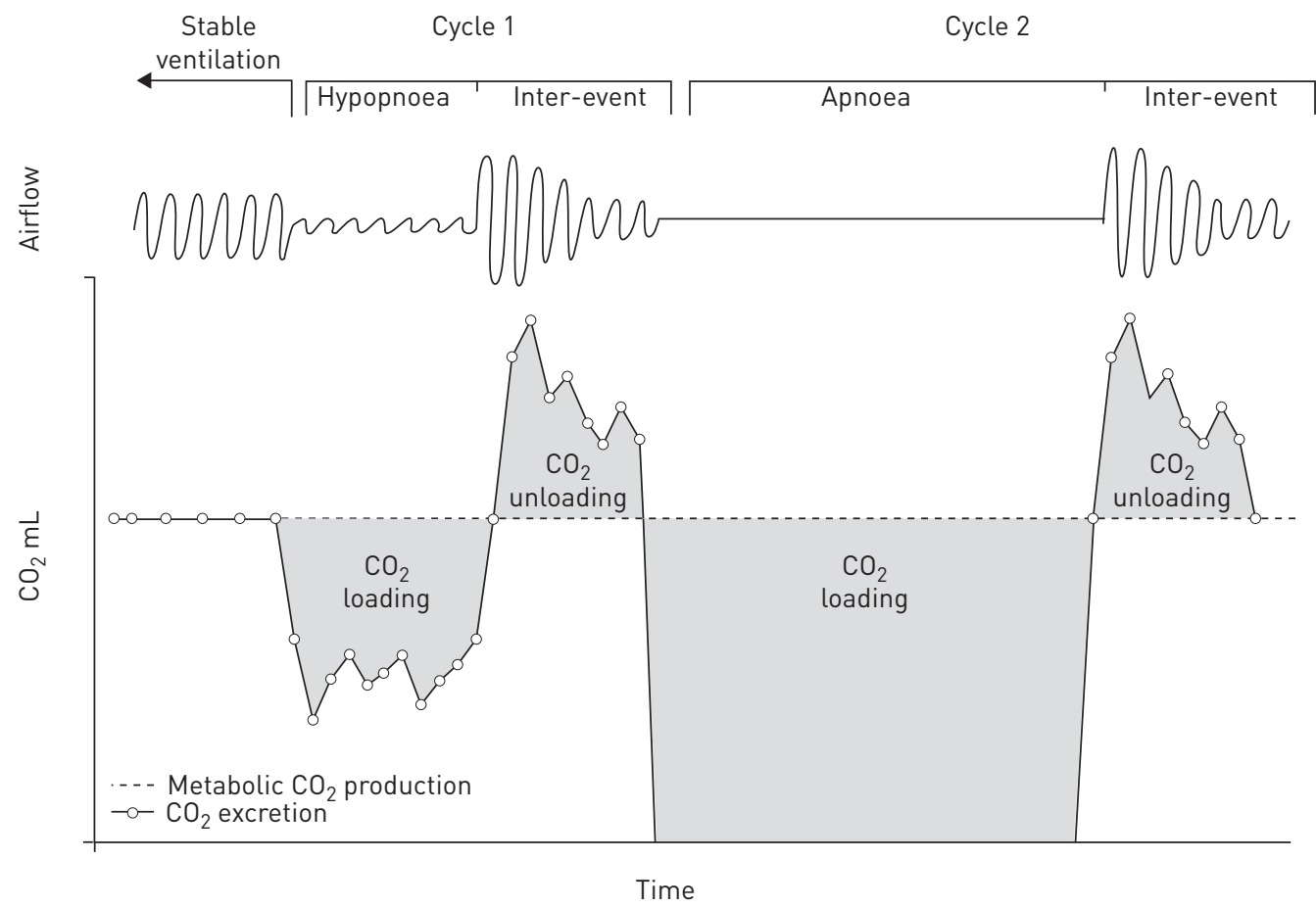

FIGURE 1 Illustration demonstrating carbon dioxide loading and unloading during short and long respiratory events. Reproduced from [23] with permission from the publisher.

of $12 \mathrm{cmH}_{2} \mathrm{O}$, increasing by $2-3 \mathrm{cmH}_{2} \mathrm{O}$ as tolerated, and an expiratory positive pressure of $5 \mathrm{cmH}_{2} \mathrm{O}$ increased by $1-2 \mathrm{cmH}_{2} \mathrm{O}$ to improve hypoxaemia or comfort. No attempt was made to titrate therapy with individual respiratory events. NIV success was regarded as avoidance of endotracheal intubation and survival for at least $24 \mathrm{~h}$ after discharge from the intensive care unit (ICU). NIV failure was defined as worsening arterial blood gas tensions on NIV resulting in intubation or death. OHS patients experienced less NIV failure (7\% versus $13 \%$ in the COPD group; $\mathrm{p}=0.037)$, fewer ICU readmissions, and lower ICU and hospital mortality. However, survival adjusted for confounders was similar at 1 year, which is unexpected. Closer observation shows that only 55\% of patients with OHS received long-term positive pressure respiratory support on hospital discharge and only $10 \%$ were prescribed NIV. This is not in accordance with consensus reports and clinical guidelines.

While long-term NIV usually corrects arterial blood gas tensions, sleepiness and vigilance and reduces cardiovascular morbidity it has become clear that deaths in this population commonly occur due to cardiovascular causes. BOREL et al. [26] examined the effects of NIV in OHS on cardiovascular, metabolic and inflammatory variables. As patients with mild OHS were included it was possible to randomise patients to NIV therapy or control group for 1 month. The authors found that while, as expected sleep and blood gas measures very significantly improved in the NIV group, inflammatory markers, endothelial function and arterial stiffness did not improve. Weight loss has been shown to improve both obstructive events [27] and REM-related hypoventilation in OHS. The extent of reversal of cardiovascular harm probably depends on the duration and extent of obesity and other genetic factors, but clearly weight loss is the goal. NIV should also be combined with all other strategies to reduce cardiovascular risk [28].

\section{Volume assured ventilation in OHS?}

Recently hybrid modes of ventilation have been developed, which aim to combine bilevel pressure support with the delivery of an assured minute or tidal volume. The underlying goal of these modes is to better adapt to the patient's own ventilatory pattern and requirements, which will clearly vary during different stages of sleep and with different activities during the day. Some devices also have an "intelligent" back-up rate and a "learn" mode in which the ventilator adapts to the patient's respiratory effort and pattern.

AVAPs (Average Volume Assured Pressure Support; Philips Respironics, Best, the Netherlands) has been used in a randomised crossover trial [29] of AVAPS versus standard pressure support in obesity hypoventilation patients and produced a small improvement on nocturnal $\mathrm{PCO}_{2}$, but no long-term quality of life improvement. In very obese patients MURPHY et al. [30] have confirmed there was no long-term 
advantage in AVAPS over optimally titrated bilevel pressure support, so these "intelligent" modes do not seem to have a role in the general population of OHS patients, although there is some evidence to suggest one of these modes (iVAPs; intelligent Volume Assured Pressure Support, ResMed, Martinsried, Germany) may aid initiation of NIV in subgroups with OHS or neuromuscular conditions who are starting NIV for the first time.

\section{Conclusions}

Chronic hypoventilation in neuromuscular disorders and OHS result from different pathophysiological processes, but both are assessed by careful attention to clinical history, identification of high risk cases and sleep study monitoring. Correction of nocturnal hypoventilation and daytime arterial blood gas tensions can be achieved using NIV, which results in relief of symptoms of nocturnal hypoventilation. However, ventilatory support is not a "cure" and it is not clear whether the cardiovascular consequences of these disorders can be reversed.

\section{References}

1 Hull J, Aniapravan R, Chan E, et al. British Thoracic Society guideline for respiratory management of children with neuromuscular weakness. Thorax 2012; 67: Suppl. 1, i1-i40.

2 Bush A. Spinal muscular atrophy with respiratory disease (SMARD): an ethical dilemma. Intensive Care Med 2006; 32: 1691-1693.

3 Ragette R, Mellies U, Schwake C, et al. Patterns and predictors of sleep disordered breathing in primary myopathies. Thorax 2002; 57: 724-728.

4 Nickol AN, Hart N, Hopkinson NS, et al. Mechanisms of improvement of respiratory failure in patients with restrictive thoracic disease treated with non-invasive ventilation. Thorax 2005; 60: 754-760.

5 Ramirez A, Khirani S, Delord V, et al. Assessment of sleep quality by pulse wave amplitude and actigraphy in children with sleep disordered breathing: evaluation at diagnosis and under non-invasive ventilation. Sleep Breath 2013; 17: 827-835.

6 Mellies U, Ragette R, Dohna Schwake C, et al. Long-term noninvasive ventilation in children and adolescents with neuromuscular disorders. Eur Respir J 2003; 22: 631-636.

7 Ward SA, Chatwin M, Heather S, et al. Randomised controlled trial of non-invasive ventilation (NIV) for nocturnal hypoventilation in neuromuscular and chest wall disease patients with daytime normocapnia. Thorax 2005; 60: 1019-1024.

8 Lyall RA, Donaldson N, Polkey MI, et al. Respiratory muscle strength and ventilatory failure in amyotrophic lateral sclerosis. Brain 2001; 124: 2000-2013.

9 Bourke SC, Tomlinson M, Williams TL, et al. Effects of non-invasive ventilation on survival and quality of life in patients with amyotrophic lateral sclerosis: a randomised controlled trial. Lancet Neurol 2006; 5: 140-147.

10 McGinley B, Halbower A, Schwartz AR, et al. Effect of high-flow open nasal cannula system on obstructive sleep apnea in children. Pediatrics 2009; 124: 179-188.

11 Chatwin M, Ross E, Hart N, et al. Cough augmentation with mechanical insufflation/exsufflation in patients with neuromuscular weakness. Eur Respir J 2003; 21: 502-508.

12 Bach JR, Ishikawa Y, Kim H. Prevention of pulmonary morbidity for patients with Duchenne muscular dystrophy. Chest 1997; 112: 1024-1028.

13 Bach J. A comparison of long-term ventilatory support alternatives from the perspective of the patient and care giver. Chest 1993; 104: 1702-1706.

14 Eagle M, Baudouin SV, Chandler C, et al. Survival in Duchenne muscular dystrophy: improvements in life expectancy since 1967 and the impact of home nocturnal ventilation. Neuromuscul Disord 2002; 12: 926-929.

15 Simonds AK, Muntoni F, Heather S, et al. Impact of nasal ventilation on survival in hypercapnic Duchenne muscular dystrophy. Thorax 1998; 53: 949-952.

16 Ishikawa Y, Miura T, Ishikawa Y, et al. Duchenne muscular dystrophy: survival by cardio-respiratory interventions. Neuromuscul Disord 2012; 2011: 47-51.

17 Soudon P, Steens M, Toussaint M. A comparison of invasive versus noninvasive full-time mechanical ventilation in Duchenne muscular dystrophy. Chron Respir Dis 2008; 5: 87-93.

18 Oskoui M, Levy G, Garland CJ, et al. The changing natural history of spinal muscular atrophy type 1. Neurology 2007; 69: 1931-1936.

19 Chatwin M, Bush A, Simonds AK. Outcome of goal-directed non-invasive ventilation and mechanical insufflation/ exsufflation in spinal muscular atrophy type I. Arch Dis Child 2011; 96: 426-432.

20 Caldarelli V, Borel JC, Khirani S, et al. Polygraphic respiratory events during sleep with noninvasive ventilation in children: description, prevalence and clinical consequences. Intensive Care Med 2013; 39: 739-746.

21 Gonzalez-Bermejo J, Perrin C, Janssens JP, et al. Proposal for a systematic analysis of polygraphy or polysomnography for identifying and scoring abnormal events occuring during non-invasive ventilation. Thorax 2012; 67: 546-552.

22 Pépin JL, Borel JC, Janssens JP. Obesity hypoventilation syndrome: an undiagnosed and undertreated condition. Am J Respir Crit Care Med 2012; 186: 1205-1207.

23 Berger KI, Goldring RM, Rapoport DM. Obesity hypoventilation syndrome. Semin Respir Crit Care Med 2009; 30: 253-261.

24 Piper AJ, Wang D, Yee BJ, et al. Randomised trial of CPAP $v$ s bilevel pressure support in the treatment of obesity hypoventilation syndrome without severe nocturnal hypoventilation. Thorax 2008; 63: 395-401.

25 Carrillo A, Ferrer M, Gonzalez-Diaz G, et al. Noninvasive ventilation in acute hypercapnic respiratory failure caused by obesity hypoventilation syndrome and chronic obstructive pulmonary disease. Am J Respir Crit Care Med 2012; 186: 1279-1285. 
26 Borel JC, Tamisier R, Gonzalez-Bermejo J, et al. Noninvasive ventilation in mild obesity hypoventilation syndrome: a randomized controlled trial. Chest 2012; 141: 692-702.

27 Buchwald H, Avidor Y, Braunwald E, et al. Bariatric surgery: a systematic review and meta-analysis. JAMA 2004; 292: 1724-1737.

28 Borel JC, Burel B, Tamisier R, et al. Comorbidities and mortality in hypercapnic obese under domiciliary noninvasive ventilation. PLoS ONE 2013; 8: e52006.

29 Storre JH, Seuthe B, Fiechter R, et al. Average volume-assured pressure support ventilation in obseity hypoventilation: a randomised crossover trial. Chest 2006; 130: 815-821.

30 Murphy PB, Davidson CH, Hind MD, et al. Volume targeted versus pressure support non-invasive ventilation in patients with super obesity and chronic respiratory failure: a randomised controlled trial. Thorax 2012; 67: 727-734. 